\title{
The concept of reliability-oriented accounting of leaks in gas supply systems
}

\author{
Olga Gnezhdilova, Anna Malysheva*, Anna Gagulina, and Aleksandr Ovsienko \\ Moscow state university of civil engineering, Moscow, Yaroslavskoye shosse, 26, Russia
}

\begin{abstract}
The concept of reliability-oriented maintenance of gas distribution networks is substantiated. Performed an analytical review of works of leading scientists in the field of the theory of gas supply of individual household. It has been established that the question of control and to prevent gas leaks remains relevant now. It is proved, that for engineering systems use gas for domestic purposes, also the greatest risk are low-intensity gas leaks, that hardly controlled by hardware due to accounting errors. An algorithm is presented for vulnerability assessment elements engineering gas supply systems for leaks. The mathematical model for the evaluation of measuring error of parametric parameter of gas flow in the system. The technique for monitoring the tightness of gas systems engineering with the rationale for selecting the method of evaluation of error results.
\end{abstract}

\section{Introduction}

The methodology of identification and selecting a failure prevention policy is aimed at mitigating the consequences of failures: reliability centred maintenance (RCM).

The RCM includes:

-identification of suitable and effective requirements for the system and preventive maintenance operations;

- detection of failures in terms of their impact on safety, technical efficiency and economical operation;

- identification of factors causing failures and mechanisms of system degradation (establishment of criteria for classifying failures as considered, critical, and so on, by type);

-evaluation of "success-failure" indicators.

Stages of RCM analysis:

- evaluation of the system design (initiation and planning);

- analysis of functional failures;

- selection of tasks, aimed at ensuring the safety of structures for personnel and the environment, efficiency and cost-effectiveness of operation.

The proposed solutions should be economically viable, safe and environment friendly.

Conducting RCM-analysis during the operation allows you to improve the methods of maintenance, to conduct constructive and other improvements.

Goals:

- Evaluation of reliability factors

\footnotetext{
* Corresponding author: MalyshevaAA@mgsu.ru
} 
- Identification of factors causing failures, and the development of measures aimed at weakening their impact on the reliability of products;

- assessment of the impact of technological processes on reliability

- making the product more reliable (including dependability) and safety;

- improvement of technical characteristics and quality of products;

- assessment and reduction of the life cycle cost of the product;

- analysis of operating conditions and their impact on the reliability of products;

- justification of the values of reliability indicators included in the technical documentation

The failure rate in the exponential distribution of mean lifetime is constant. Taking into account a certain tendency in measurement of failure rate of products, a failure time distribution can be described by Weibull distribution.

Exponential distribution requires data on the number of failures to be taken into account and the value of accumulated lifetime of the tested samples;

Weibull distribution-it is required to know the lifetime of non-recoverable products for the numerical method; for the graphical method-the data of observations of product time to failure are required.

The reliability of energy systems, which includes the gas supply system, is a complex indicator and is defined by a set of private indicators. In relation to natural gas distribution systems, the main of them is the accounting of energy consumption.

Gas engineering systems are a set of elements that supply gas to the source of consumption, functionally providing the required pressure, quality and safety of the system as a whole. Normative documents require gas distribution organizations (GDO) to conduct mandatory energy surveys in relation to the technical process: gas transportation through pipelines, maintenance and operation of gas distribution networks [1-3]. The practical objectives of the energy survey are to obtain reliable information on the use of fuel and energy resources and to develop measures for energy saving and energy efficiency.

For gas distribution organizations, target indicators for energy saving and energy efficiency have been set, which should be ensured during the implementation of energy saving and energy efficiency programs for GDO.

As an example, the resources of one of the companies serving gas distribution networks with a length of more than $300000 \mathrm{~km}$ are considered. Their complex ensures the operation of about 300 thousand gas reduction points.

In the structure of the fuel and energy balance of GDO, natural gas consumption prevails (almost 90\%). Its technological losses, in turn, according to the reported data are close to $80 \%$ of its total consumption. Evidently, in this situation, the determination of the actual gas losses and the search for ways to reduce them are the highest priority for improving the energy efficiency of the GDO which can be achieved by introducing telemetry systems for key target parameters. Given that the number of distribution points increases every year, out of 34700 gas control points, only $18 \%$ are equipped with a telemetry system. At the same time, it has been established, that the introduction of target indicators sets a relative decrease in specific gas losses during its transportation through distribution networks for various GDO ranging from 1-3 to $12-20 \%$ [6].

\section{Theoretical research. Methods and techniques}

It is considered, that whatever the technical condition of the gas distribution networks, the value of gas losses is unchanged and depends on the geometric volume of networks, gas pressure and leakage coefficient. There is no normalized value for this indicator. The paper proposes taking into account the real technical condition of gas pipelines for calculating the target indicator. 
The existing analysis scheme, which allows to estimate the load and resource supply of gas pipelines of each pressure category of the entire gas distribution network as a whole, is not suitable for efficiency increasement, as we receive generalized indicators showing the presence or absence of problems in a particular network. The objective need to switch to a personalized analysis of the gas distribution network load has appeared, with objective detalization, to develop an algorithm for analyzing the load of networks, taking into account the property appurtenance for the formation of forecast investment programs.

The next step of analytical research was the assessment of the level of import substitution of equipment and components. A bottleneck has been identified, which is the equipment of gas reduction points, in particular, the gas pressure regulator in terms of its characteristics, manufacturing quality and reliability of operation, as well as the associated service frequency of domestic production are significantly inferior to Italian and German manufacturers.

Additionally, the issue of implementation of telecontrol system (TCS) is considered. The introduction of these systems allows to adjust the frequency of maintenance and reduce the frequency of scheduled operations which is not yet regulated.

Structurally, all elements of engineering systems have different types of connections. Their technical requirements provide for complete hermiticity throughout the period of operation.

In practice, it often happens that the hermiticity is broken and gas is leaking into the surrounding space. Such leaks and related factors lead to dangerous situations, gas poisoning, fire and explosions.

Element components of gas distribution engineering systems, according to their quality and functional characteristics should have a high level of reliability throughout the whole operating period.

Information about the level of reliability and intensity of parametric change for the resource period for a significant part of the elements of engineering systems is not given in the technical characteristics provided by the manufacturer. The operational documentation contains instructional requirements for normal operating conditions and the frequency of service actions to maintain efficiency.

To eliminate the identified defects, according to standard methods, an assessment of reliability indicators is carried out, according to which the correlation field of changes in the reliability of the system elements from the period of their operating time.

Management of gas supply and gas distribution networks is carried out through control systems, which ensures optimal conditions of technological processes in the energy sector. In the scope of the country, effective accounting of gas consumption and improvement of the design of metering devices which provides a reduction in measurement error by at least $1 \%$ will allow to obtain a multimillion-dollar economic effect.

Gas pressure regulators are devices that serve to automatically maintain the pressure at a given level. Depending on the required pressure (the location of the controlled point in the gas pipeline), the pressure regulators are divided into "before" and "after" regulators, which are typical for regulators installed in gas distribution points. According to the principle of operation, the regulators are divided into direct-flow and combined.

An automatic pressure regulator consists of an actuator and a regulator. The main part of the actuator is the sensing element, which compares the signals of the set point device and the current value of the regulated pressure. The actuator converts the command signal to the regulating action and to the corresponding movement of the movable part of the regulator due to the energy of the operating environment. If the permutation effort developed by the sensitive element of the regulator is high enough, then it itself performs the functions of controlling the regulatory body. Such regulators are called self-actuated controllers. 
Monitoring of gas pressure in gas pipelines is conducted by measuring it during the period of the highest consumption (the winter period) and during the hours of maximum gas consumption. Technologically, this procedure is carried out as follows: unscheduled pressure measurements are made to clarify the effective radius of existing gas distribution plants, the possibility of connecting new consumers is identified. Pressure measurements are made at pre-planned points of the gas network, at the exit from gas distribution plant and at consumers according to a scheme approved by the technical management of the operating organization in the prescribed manner. The points of pressure measurement on gas pipelines are determined by the operating organization on the basis of operating experience, taking into account applications from consumers for gas pressure reduction. The measurement scheme includes points on gas pipeline sections most distant from the gas distribution plants (along the gas flow) consumers and other spots unfavorable in terms of gas supply. If the pipeline plugging by hydrate and condensate plugs is detected, additional measurements are made. To measure the pressure on gas pipelines manometers of various types are used.

To characterize the power of gas pipelines, the gas flow rate is set. In practice, during the assessment of the efficiency of the gas supply network, it is necessary to know not only the consumption of gas supplied to the consumer, but also its correlation to the time factor: day, month, year, which will create a statistical basis for economic accounting of reciprocal payments between suppliers and consumers of gas fuel. In gas consumption systems, various devices are used to account for gas consumption. To measure the flow rate of gas flow meters are used, and to account for the mass and volume - counters. Manufacturers offer a wide range of combined devices: flow meter with a counter. Standard flow meters of pressure ratio are classified into: electromagnetic, turbine, vortex, ultrasonic.

For domestic purpose gas engineering systems, where the flow pressure is low, it is rather difficult to establish leaks due to the high dispersion of points of vulnerability, also the most dangerous are sluggish gas leaks, which are difficult to fix by hardware due to metering errors.

It is established, that in engineering systems, there are points of vulnerability in terms of gas leakage. It is generally accepted that the cross-sectional area through which the gas is leaked does not exceed $1 \%$ of the cross-sectional area of the pipeline.

It is proposed to carry out a ranking to determine the level of danger and the probability of a gas leak, introducing the coefficient of vulnerability and the uncertainty value of the point by the magnitude of the error, using data from manufacturers of engineering gas supply and transmission equipment.

The analysis of typical gas leakage points is presented in Table 1 [7-8].

Table 1 Characteristic groups of gas loss sources and their number.

\begin{tabular}{|c|l|c|}
\hline № & \multicolumn{1}{|c|}{$\begin{array}{c}\text { The name of the characteristic group of gas } \\
\text { losses sources }\end{array}$} & $\begin{array}{c}\text { The number of loss } \\
\text { sources, } Q, \mathrm{~m}^{3} / \mathrm{h}\end{array}$ \\
\hline 1. & $\begin{array}{l}\text { Gas distribution plant and block gas } \\
\text { distribution plant }\end{array}$ & 1514 \\
\hline 2. & $\begin{array}{l}\text { Boxed gas distribution plant with a capacity of } \\
\text { more than } 50 \mathrm{~m}^{3} / \mathrm{h}\end{array}$ & 3091 \\
\hline 3. & $\begin{array}{l}\text { Boxed gas distribution plant with a capacity of } \\
\text { less than } 50 \mathrm{~m}^{3} / \mathrm{h}\end{array}$ & 617 \\
\hline 4. & $\begin{array}{l}\text { Plug valves } \\
\text { Union and spigot joints on the linear part of } \\
\text { the gas pipeline, plugs, etc. }\end{array}$ & 202267 \\
\hline 6. & Gate valves and rectifiers & 19182 \\
\hline
\end{tabular}




\begin{tabular}{|c|l|c|}
\hline 7. & $\begin{array}{l}\text { Flanged joints, insulating flange joints, } \\
\text { expansion joints (bellows or lens), condensate } \\
\text { traps, valves, etc. }\end{array}$ & 51938 \\
\hline 8. & Gas metering stations & 345 \\
\hline 9. & Stabbing valves & 18759 \\
\hline & Total: & 439095 \\
\hline
\end{tabular}

The question of choosing a rational method for solving the problem of static evaluation of the functioning of engineering gas systems and the maximum similarity of the model remains open.

Since this problem is relevant, many scientists and manufacturers are trying to solve the problem, offering different options and techniques $[9,10]$.

Theoretically, this problem is solved by the matrix method, which establishes the relationship between the errors in the components of the column of free terms $\|A\| \cdot\left\|A^{-1}\right\|$ and the solution error $\xi_{Y, b}$, i.e. estimated values:

$$
\left\|\xi_{Y}\right\| \leq\|A\| \cdot\left\|A^{-1}\right\| \cdot\left\|\xi_{b}\right\|
$$

In turn, the matrix has $\lambda$ a maximum and a minimum of eigenvalues expressed:

$$
\|A\| \cdot\left\|A^{-1}\right\|=\left|\frac{\lambda_{\max }}{\lambda_{\min }}\right|
$$

Using the basic models for solving matrices by additional transformations, we obtain an expression allowing us to estimate the errors of the results by the quality of the original data.

Representing a given matrix $\|A\| \cdot\left\|A^{-1}\right\|$ by the ratio $\operatorname{det}(A)=\prod_{i=1}^{n} \lambda_{i}$ we get the desired expression:

$$
\operatorname{det}(A) \leq \lambda_{\max }^{n}\left(\frac{\lambda_{\max }}{\lambda_{\min }}\right) \leq n^{n / 2}\left(\frac{\lambda_{\min }}{\lambda_{\max }}\right) \operatorname{det}(A)=\prod_{i=1}^{n} \lambda_{i}
$$

When developing an algorithm for monitoring the hermiticity of engineering gas systems, it is necessary to define the characteristics of the receiving device in order to ensure optimal signal conversion $X_{n}$. For this purpose, it is assumed that in each situation the probability density of the observed signals $P_{i}\left(X_{n} \mid \alpha_{i}\right)$, is determined, with an accuracy of the environmental parameters represented by the vector $\alpha_{i}$. The values $P_{i}\left(X_{n} \mid \alpha_{i}\right)$ represent a set of minimally sufficient statistics for derived loss coefficients and accepted probabilities.

The theoretical aspects of gas flow accounting are subjected to different distribution laws, in particular, when establishing the regularities of relations between the processes occurring in gas distribution systems depend on many, among which there are unknown, and in some cases often uncontrollable factors. 
Theoretically, the parametric relationship is given by variables $x$ and $y$, the density of which is not always clearly defined, because one of the values of the variable $x$ can correspond to the set of values of the variable $y$.

With a sufficient degree of accuracy, it can be argued that for the parametric characteristics of performance indicators of gas consumption systems, the correlation function $y=f(x)$ is applicable in which each value of $x$ corresponds to a statistical series of distribution $y$.

It is assumed that the relationship between $\mathrm{x}$ and $\mathrm{y}$ is probabilistic (stochastic).

For gas consumption parameters, the regression equation takes the form:

$$
Q=f(p)
$$

where $Q$ is the gas flow rate; $P_{\text {у.г. }}-$ probability of leakage $\mathrm{m}^{3} / \mathrm{h}$.

The analysis involves the establishment of the relationship between the different values of influence, their significance, reliability and adequacy of the obtained measurement values.

\section{Efficiency of assessment}

Experimental studies of gas leakage estimates are performed by obtaining $\mathrm{n}$ - independent measurements of $Q_{i}$, values of constant magnitude, parameters such as pressure, gas volume and flow rate in a pipeline. To establish the measurement accuracy, the deviation of the result from the average value is established, the deviation value is represented with an indicator of the random deviation $\delta i$.

$$
Q_{n i}=\bar{Q}_{n}+\delta_{n_{i}}
$$

To establish the arithmetical mean of the measurement result, converging in probability to the average value $\bar{Q}_{n}$, with any probability distribution law of the measurement result is an independent point estimate of the average value:

$$
M\left(\frac{1}{n} \sum_{i=1}^{n} Q_{i}\right)=\frac{1}{n} \sum_{i=1}^{n} M\left(Q_{i}\right)=\frac{1}{n} \sum_{i=1}^{n}\left[M(\bar{Q})+M\left(\delta_{i}\right)\right]=\bar{Q}
$$

The resulting equation of expression shows that the accuracy of a multiple measurement result depends on the effectiveness of estimate of the average value.

The standard deviation of the measurement result is determined from the expression:

$$
S_{Q}=\sqrt{\frac{1}{n-1} \sum_{i=1}^{n}\left(Q_{i}-\overline{Q_{n}}\right)^{2}} .
$$

The optimal is a function in which the least-squares condition is satisfied:

$$
\sum_{i=1}^{n}\left(Q_{i}-\overline{Q_{n}}\right)^{2}=\min
$$

The analysis involves the establishment of the relationship between the different values of influence, their significance, reliability and adequacy of the obtained measurement values. 
Using a standard technique, a correlation field of factor influence is constructed. For variable factors, the relationship can be established using the n-dimensional space in equation of second order:

$$
y=b_{0}+\sum_{1}^{n} b_{i} x_{i}+\sum_{1}^{n} b_{i j} x_{i} x_{j}+\sum_{1}^{n} b_{i i} x^{2}{ }_{i},
$$

where $y$ is the function of the target (response) of multifactor variables; $x_{\mathrm{i}}$ - independent factors; $b_{\mathrm{i}}$ - regression coefficients; $b_{\mathrm{ij}}$ - coefficients characterizing the double effect of factors $x_{\mathrm{i}}$ и $x_{\mathrm{j}}$ on the objective function.

The criterion for the proximity of the correlation (between $x$ and $y$ ) to the linear functional relationship is the correlation index

$$
r=\frac{n \sum x_{i} y_{i}-\sum x_{i} \sum y_{i}}{\sqrt{\left(n \sum x_{i}^{2}-\left(\sum x_{i}\right)^{2}\right)}\left(n \sum y_{i}^{2}-\left(\sum y_{i}\right)^{2}\right)},
$$

where $n$ is the number of measurements.

The value of the correlation coefficient $\mathrm{r}$ is always less than one. When $r=1$, the relationship between $\mathrm{x}$ and $\mathrm{y}$ becomes functional, usually consider the tightness of the relationship satisfactory, if $r>0.5$, good - at $r=0.8 \ldots 0,85$.

In addition, the concept of the determination coefficient $K_{d}=r^{2}$, characterizing the variability of parameters is introduced.

The calculations use the estimation of factor influence and the level of validity. Thus, if according to the experiments $r=0.9$, then $K_{d}=0,81$, i.e. the variability of the function in $81 \%$ due to the parameter $x$, and the influence of other factors is estimated $19 \%$ [11].

\section{Discussion}

It should be noted that a two-variant, non-alternative hypothesis representing extreme events is considered: the engineering gas system is hermetic and there is a leak. The justification for this is the factor danger of the event - there is a leak and the further task of eliminating the negative consequences, starting with unpleasant sensations in the form of foreign smell, ending with an explosion with catastrophic consequences for humans, structures and the environment [12-14].

\section{Conclusion}

The analytical review of works of the leading scientists in the field of the theory of gas supply is completed.

It is established, that the issue of control and prevention of gas leaks remains relevant at the present time.

It is proved that for engineering systems of domestic gas usage, where the flow pressure is low, it is difficult to establish leaks due to the large dispersion of vulnerability points, also the most dangerous are sluggish gas leaks, the presence of which is difficult to be controlled by hardware due to the presence of accounting errors.

An algorithm for assessing the vulnerability of elements of the engineering system of gas supply by the leakage parameter is presented.

A mathematical model for estimating the parametric error of the gas flow parameter in the system is developed. 
The method of monitoring the tightness of engineering gas systems with the justification of the choice of the method of evaluation of the error of the results is proposed.

Research is conducted in the framework of the implementation of the GBS No. 13.11847.2018/11.12 on the theme "Development of a model for operational management of urban gas distribution systems based on the principle of regulation by disturbance".

\section{References}

1. M. Aboubacar, J. P. Aguayo, P. M. Phillips, T. N. Phillips, H. R. Tamaddon, B. A. Snigerev and M. F. Webster J. Non-Newtonian Fluid Mech. 126 207-220 (2005)

2. I. V. Morenko and A. Mazo J. High Speed Hydrodynamics. Second International Summer Scientific School. Cheboksary, Russia. 307- 311 (2005)

3. I. A. Bublichenko, B. E. Mokhnonozhkin J. Instruments and Experimental Techniques 5(42) 697-700 (1999)

4. V. E. Drejzin, P. Borzenkov J. News South West State University 42-50 (2015)

5. V. Melkumov, G. A. Kuznecov, M. J. Panov J. Scientific Herald Voronezh GUS. Construction and architecture 4(28) 32-40 (2012)

6. S. V. Vlasichev J. Gas Russia 4 4 8-11 (2014)

7. O. A. Gnezdilova, A. M. Krygina, A. N. Kobelev J. Industrial and civil stroitelnstvo 10 (2009)

8. O. A. Gnezdilova, N. S. Kobelev, M. Aleynikov J. Research Bulletin VGASU. Construction and architecture 1(13) (2009)

9. S. A. Sazonova J. Information technologies in construction, social and economic systems 4 (14) 16-23 (2018)

10. P. D. Kapyrin and N. S. Sevryugina IOP Conf. Ser.: Mater. Sci. Eng. 451012192 (2018)

11. P. D. Kapyrin and N. S. Sevryugina IOP Publishing Ltd 365042018 (2018)

12. A. M. Sergeev, A. V. Kim, V. B. Gildenburg, M. D. Chernobrovtseva, V. I. Pozdnyakova, I. A. Shereshevskii, M. Lontano J. Laser and Particle Beams 1(17) 129-138 (1999)

13. Yang H., Ge C., Ye H., Wang G. J. Gaojishu Tongxin 6(20) 628-631 (2010)

14. A. I. Kolosov, G. A. Kuznetsova, O. A. Gnezdilova J. Russian Journal of Building Construction and Architecture 2 (38) 19-26 (2018) 